\title{
OPTIMIZATION ANALYSIS OF ENERGY-ABSORBING STRUCTURES IN SUBWAY TRAIN
}

\author{
Bingzhi Chen, ${ }^{*}$ Ruixian Qin, ${ }^{*}$ and Chaozhe Jiang**
}

\begin{abstract}
An energy-absorbing structure from the front end of subway vehicle was studied to promote crashworthiness during a collision by increasing energy-absorption and decreasing collision force in this paper. A size optimization problem was solved using the non-linear programming by quadratic Lagrangian algorithm by integrating collision simulation code PAM-CRASH in the multidiscipline optimization platform Isight. Moreover, the energy-absorbing structure was redesigned using the topology optimization in OptiStruct module to promote its deformation efficiency. It is indicated that combination of size and topology optimization techniques aforementioned is feasible and efficient in promoting the crashworthiness performance of energy-absorbing structure and providing more safety allowance for passengers.
\end{abstract}

\section{Key Words}

Subway vehicle, energy-absorbing, crashworthiness analysis, structural optimization

\section{Introduction}

In the past few years, the subway traffic transportation pattern has been adopted in increasing numbers of cities because of its considerable carrying capacity and quite convenience. Structural safety has been given enough attention to ensure that the damage from collision accident could be decreased to the lowest limit. It is of great significance to study passive safety technology in preventing losses of casualties and properties during a collision by effective crashworthiness design and energy-absorption protection system. It is widely accepted that thin-walled structures tend to absorb much collision energy and decrease the impact acceleration pulse by structural plastic

* School of Traffic and Transportation, Dalian Jiaotong University, People's Republic of China; email: chenbingzhi06@ hotmail.com

** School of Transportation and Logistics, Southwest Jiaotong University, Chengdu, People's Republic of China; University of Waterloo, Waterloo, Ontario, Canada; e-mail: jiangchaozhe@ swjtu.cn, chaozhe.jiang@uwaterloo.ca

Recommended by Prof. Anmin Zhu

(DOI: 10.2316/Journal.206.2017.3.206-4983) deformation. The familiar energy-dissipation components used in railway vehicles include circle tube, square tube, tapered tube; especially, the square tube is widely adopted as its energy-absorbing stability. What is difficult in designing the energy-absorption structure is how to determine the size of each part of energy-absorption device, the tubes with small thickness tend to bring the deformation into instability and low energy absorption, while large thickness leads to a high initial collision pulse which makes passenger feel uncomfortable. So, it is necessary to determine the reasonable thickness for each component of energy-absorption device in railway vehicle.

Lots of criteria in evaluating crashworthiness for railway vehicles have been put forward in some general standards such as EN15227 [1] and GM/RT2100 [2]. It is proposed that the collision force does not exceed a limitation of $3,000 \mathrm{kN}$ in GM/RT2100 standard. Belytschko first succeeded in the car collision analysis by adopting shell element and the explicit time integration. At present, the structural crashworthiness promotion is a critical issue in the safety field of rail vehicles, particularly with a gradual increase in operational speed [3].

The issues involving optimization analysis to improve crashworthiness have been increasingly concerned and studied. Kurtaran et al. presented an integration methodology of optimization methods, FE simulations and approximation methods for crashworthiness design optimization [4]. Zarei and Kroger realized the maximization of the energy absorption and specific energy absorption for tubes by adopting the multi-objective optimization technique and the response surface method [5]. Xie et al. enhanced the crashworthiness of vehicle by increasing the specific energy absorption of the front-end structure with a modified method of feasible directions model [6]. Gu et al. solved a deterministic multi-objective optimization problem by combining design of experiment with non-dominated sorting genetic algorithm II based on the metamodels [7].

To maximize the energy absorption for a thin-walled structure under the constraint condition of the impact load, Jiazhi et al. optimized its rectangular section by using the response surface method considering the crashworthiness [8]. Lanzi et al. had the shape optimized for a 
conical absorber with elliptical cross-sections by adopting response surfaces and genetic algorithms and create a highefficiency structures integrated with lightweight and better absorption capabilities [9]. Sun et al. adopted the kriging modelling technique in robust optimization to promote the crashworthiness performance of a foam-filled thin-walled structure [10].

Mrzygłód and Kuczek proposed a new crashworthiness concept in the optimization problem of car bodies for high-speed trains to provide more safety redundancies for the passengers, which also realized the lightweight of the structure under stress constraints [11]. Kim et al. applied topology optimization for shell frame of leading-car structure to enhance crash energy absorption in the conceptual design phase [12]. Pedersen had researched on for the way of obtaining a desired energy absorption history with topology optimization to promote the crashworthiness of vehicles [13]. Mayer et al. had the crash energy absorption maximized by adopting the topological optimization for a given volume of components with homogenization method and optimality criteria [14]. Pedersen obtained a desired energy absorption history by using the topology optimization for a structure created by 2D-beam elements [15]. Avalle et al. achieved a best identification of geometrical configuration for an energy-absorbing device by using an iterative algorithm based on a multi-point approximation scheme and the steepest descent method [16]. Spath et al. carried out an integration by combining topology and shape optimization approaches and described the potential of the structure optimization [17].

Based on multivariable broken line seams, Zhang et al. obtained the optimal motion planning for welding a robot [18]. Chen et al. investigated multi-objectives optimum design of parallel conveyor by considering maximum load, energy and performance [19]. Parameter optimization was widely adopted in improving the structural performance, Liang et al. obtained the optimal structural parameters of exterior spiral for a novel spiral in-pipe robot [20].

This work mainly focussed on the optimization problem of energy-absorbing structure for the subway vehicle from two aspects. First, the size optimization of two-level energy-absorbing structure was conducted using the integration platform Isight and explicit dynamical analysis code PAM-CRASH. Second, further promotion of crashworthiness performance was realized for energy -absorbing structure based on the results of size optimization, the load transfer path was redesigned by the topology method in Optistruct.

\section{Basic Theory}

\subsection{Large Deformation Dynamic Analysis Method}

The main theories adopted in numerical analysis for large deformation dynamic analysis of vehicle structures are described as the following formulas under the Lagrangian description in the $(x, y, z)$ coordinate system:

$$
\sigma_{i j, j}+\rho f_{i}=\rho \ddot{x}_{i}
$$

where $\rho, \sigma_{i j}, f_{i}$ and $\ddot{x}_{i}$ are the density, the stress tensor, the bulk force and the acceleration from current configuration, respectively $(i, j=x, y, z)$

$$
\rho \gamma=\rho_{0}
$$

where $\gamma$ is the relative volume and $\rho_{0}$ is the initial density.

$$
\dot{E}=V S_{i j} \dot{\varepsilon}_{i j}-(p+q) V
$$

where $\dot{E}, \dot{\varepsilon}_{i j}, q$ and $V$ represents the current energy, the rate of strain tensor, the viscous resistance and the current volume, respectively.

$$
\begin{gathered}
S_{i j}=\sigma_{i j}+(p+q) \sigma_{i j} \\
p=-\sigma_{k k} / 3-q
\end{gathered}
$$

where $S_{i j}$ is the deviatoric stress and $p$ is the pressure.

The equilibrium equation of structure can be expressed in the Galerkin form:

$$
\begin{gathered}
\int_{V}\left(\rho \ddot{x}_{i}-\sigma_{i j, j}-\rho f_{i}\right) \delta x_{i} d V+\int_{S^{0}}\left(\sigma_{i j}^{+}-\sigma_{i j}^{-}\right) n_{j} \delta x_{i} d S \\
+\int_{S^{1}}\left(\sigma_{i j} n_{j}-t_{i}\right) \delta x_{i} d S=0
\end{gathered}
$$

Formula (4) can be educed as follows by considering the divergence theorem:

$$
\int_{V}\left(\sigma_{i j} \delta x_{i}\right)_{, j} d V=\int_{S^{0}}\left(\sigma_{i j}^{+}-\sigma_{i j}^{-}\right) n_{j} \delta x_{i} d S+\int_{S^{1}} \sigma_{i j} n_{j} \delta x_{i} d S
$$

The equation in discretization form using the finite element method is given as follows:

$$
M \ddot{x}(t)=P(x, t)-F(x, \dot{x})
$$

where $M, \ddot{x}(t)$ and $P$ represents the mass matrix, the nodal acceleration vector and the load vector, respectively. The explicit central difference method is widely applied for numerical analysis. The numerical analysis for energyabsorbing structure was carried out in PAM-CRASH based on the theory mentioned above.

\subsection{NLPQL Algorithm}

The NLPQL (non-linear programming by quadratic Lagrangian) algorithm [21] is a kind of sequential quadratic programming, which is for solving nonlinearly constrained problems with differentiable objective and constraint function. It is mathematically described as follows:

$$
\begin{array}{ll}
\min & f(x) \\
\text { s.t } & g_{i}(x)=0, \quad i=1, \ldots, m, \quad x \in R^{n} \\
& g_{j}(x) \geq 0, \quad j=1, \ldots, n \\
& x_{L} \leq x \leq x_{U}
\end{array}
$$




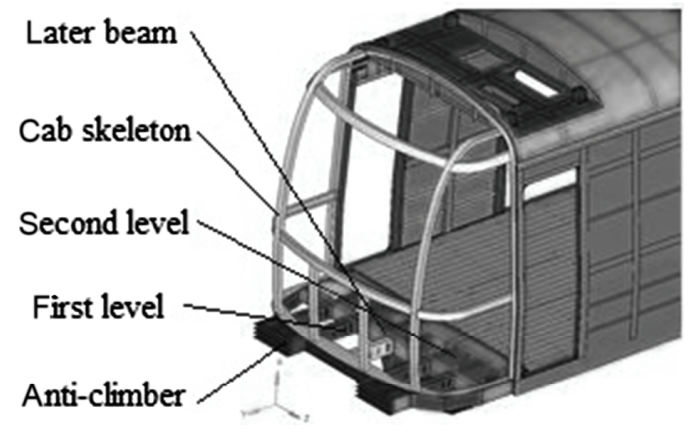

Figure 1. The local view of finite element model for the end structure.

where $f(x)$ is the objective, $g_{i}(x)$ is the equality constraints and $g_{j}(x)$ is the inequality constraints, $x_{L}, x_{U}$ are, respectively, lower limit and upper limit of design variables. The following size optimization for energy-absorbing structure is based on the NLPQL algorithm.

\subsection{Topology Optimization Method}

The topology optimization technique is a new method to solve optimal material distribution in certain structure under any load. In topology optimization, the SIMP (simplified isotropic material with penalization) approach, first proposed by Bendsøe in 1989 [22], is the most popular density variable relaxation method and adopted in OptiStruct, which achieves wide application in many engineering areas. The relation between the relative density and the material property is given by power law as follows:

$$
E\left(\rho_{i}\right)=\rho_{i}^{p} E_{0}
$$

where $p$ is the penalization parameter, $E_{0}$ is Young's modulus of material and $\rho_{i}$ is the relative density.

\section{Size Optimization for Energy Absorption Structure}

\subsection{Finite Element Model}

A finite element model suitable for crash analysis was established with an element size of $25 \mathrm{~mm}$ and 870,793 elements are generated, of which the focussed location was refined with much smaller mesh size. The weight of vehicle under normal state is $32 \mathrm{t}$. The finite element model of front-end structure for energy-absorbing is shown in Fig. 1, it consists of two anti-climber modules, first level (including five regular tubes) and second level (including six regular tubes) and lateral beam.

\subsection{Size Optimization Model}

With the maximum of the energy absorption $(E)$ by the energy-absorbing tubes and the minimum of collision force $(F)$ as the objective function, the thicknesses $\left(T_{1}, T_{2}\right)$ of the two-level energy-absorbing tubes were determined

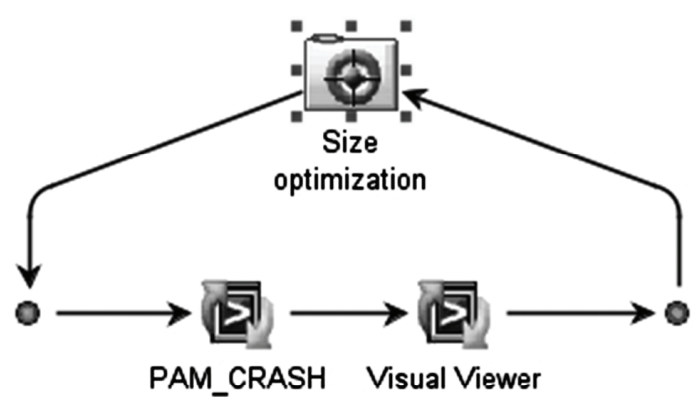

Figure 2. Data flow for optimization.

as the design variables. The multi-objective optimization model is described as follows:

$$
\begin{aligned}
& \max E\left(T_{1}, T_{2}\right) \\
& \min F\left(T_{1}, T_{2}\right) \\
& \text { s.t. } 1.0 \leq T_{1} \leq 4.0 \\
& 4.0 \leq T_{2} \leq 10.0
\end{aligned}
$$

The thickness of first-level tubes in the initial design is $2.0 \mathrm{~mm}$ and the second is $8.0 \mathrm{~mm}$, which was input as the initial value of optimization problem described above.

The optimization process established in Isight is shown in Fig. 2, explicit analysis code PAM-CRASH and its visual postprocessor were integrated to generate a dataflow cycle. The solution module PAM-CRASH and postprocessing module Visual Viewer were drove by two batch files automatically in Isight, which was used for generation and extraction of results such as energy absorption and peak force. During each iteration, the response information was transferred to Isight files management and the design variables were updated based on the change of response value, new design variables were assigned to input file *.pc, PAM-CRASH was called subsequently by batch file for analyzing updated input file. The output data of energy absorption and collision force were delivered to Isight for new assessment.

\subsection{Analysis Results}

A fully loaded car was set to crash towards a rigid wall at a speed of $25 \mathrm{~km} / \mathrm{h}$, of which the simulation result was prepared to be integrated for size optimization, including energy absorbed by two-level energy-absorbing tubes and collision force.

The iteration histories of design variables $T_{1}, T_{2}$ and objectives $E, F$ are shown in Fig. 3, the abscissa axis represents iteration cycles. For the last iteration, optimization process stopped with convergence after 27 cycles. The optimal thicknesses of the two-level energy-absorbing tubes are $3.56 \mathrm{~mm}\left(T_{1}\right)$ and $4.0 \mathrm{~mm}\left(T_{2}\right)$, respectively. There is an obvious increase of total energy absorption from 584.5 to $922.6 \mathrm{~kJ}$, and the maximum collision force decreases from 2,908 to $2,477 \mathrm{kN}$.

Deformation comparison of energy-absorption structure at the end of vehicle is shown in Fig. 4, where the 


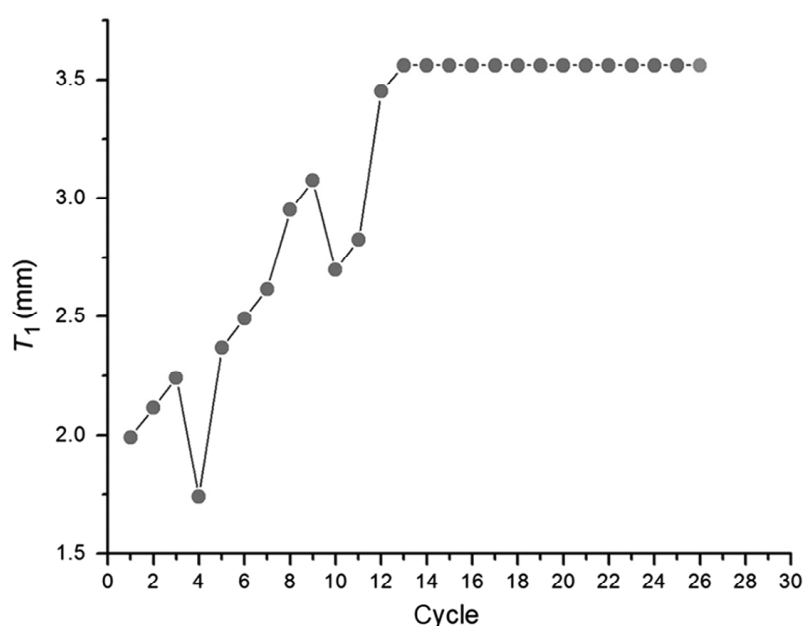

(a)

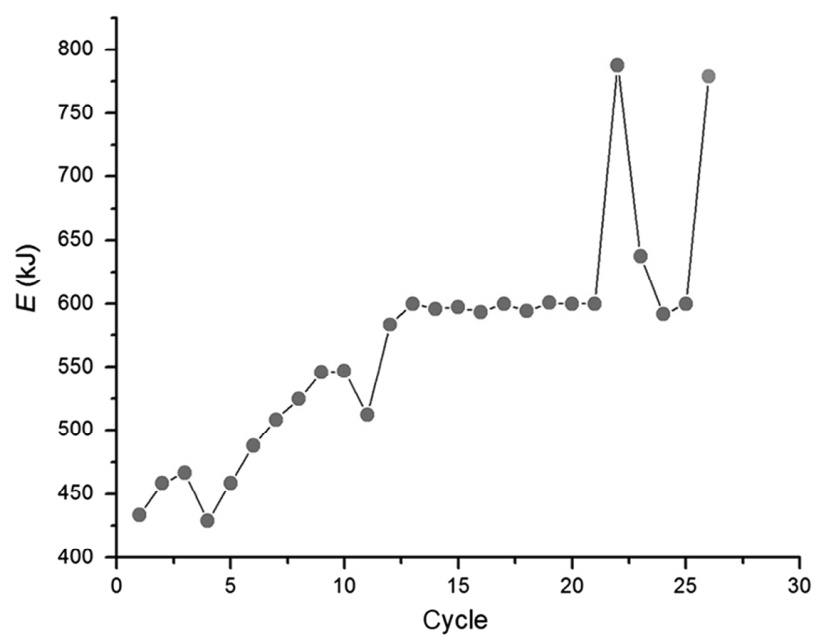

(c)

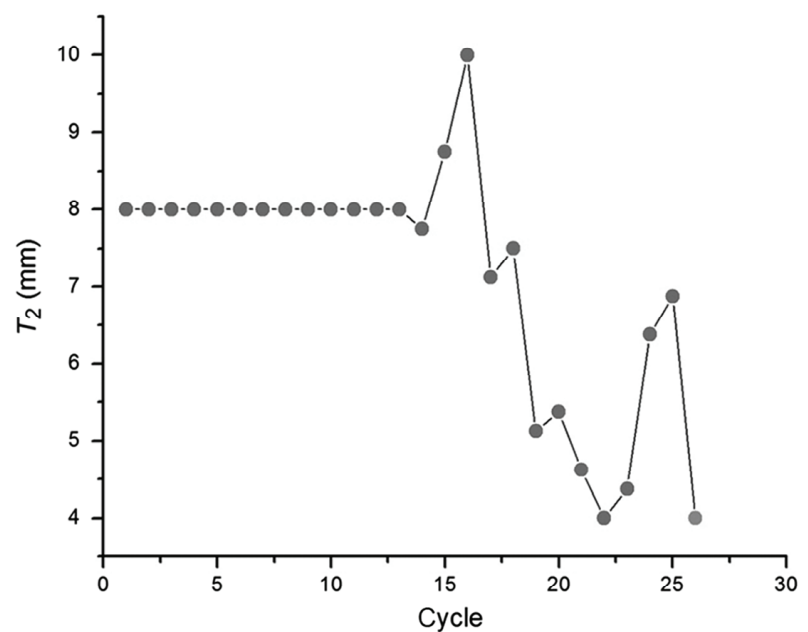

(b)

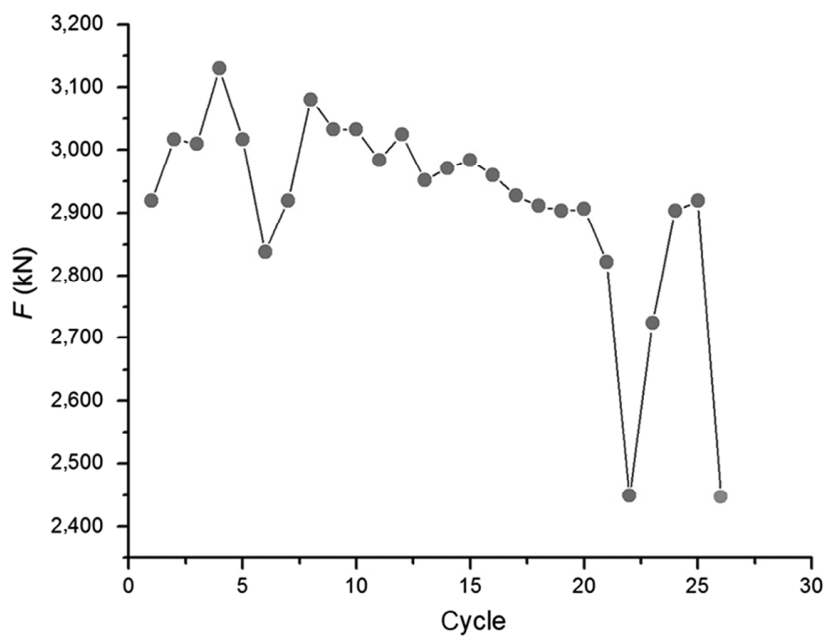

(d)

Figure 3. Iteration history of $T_{1}(\mathrm{a}), T_{2}(\mathrm{~b}), E(\mathrm{c})$ and $F(\mathrm{~d})$ versus iteration cycles.
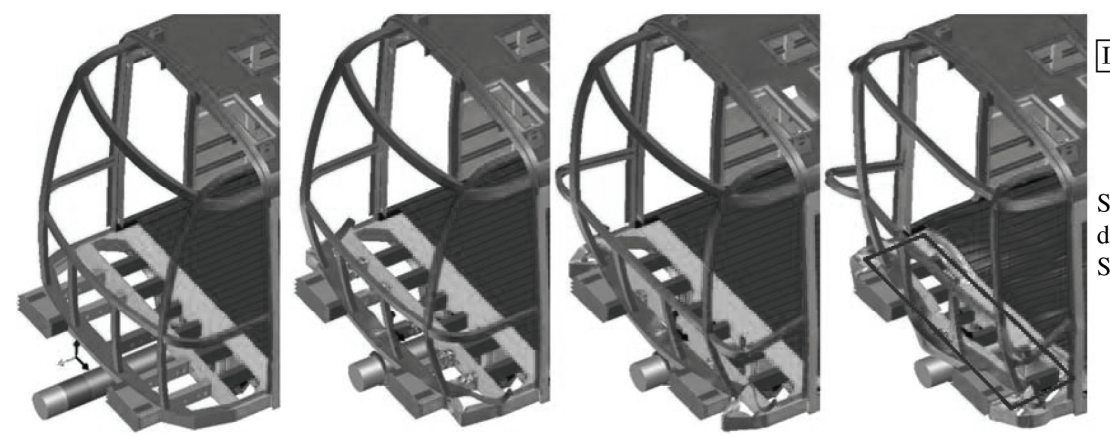

Initial

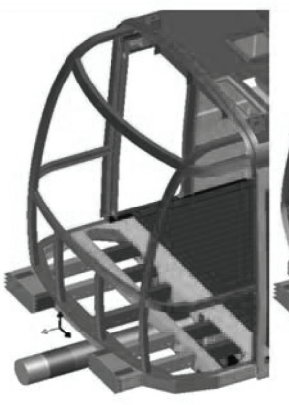

$0 \mathrm{~ms}$

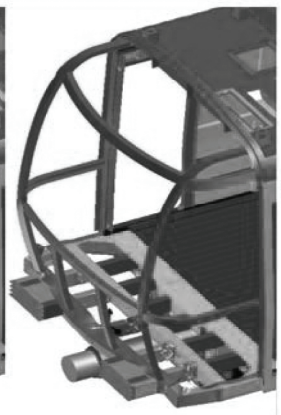

$80 \mathrm{~ms}$

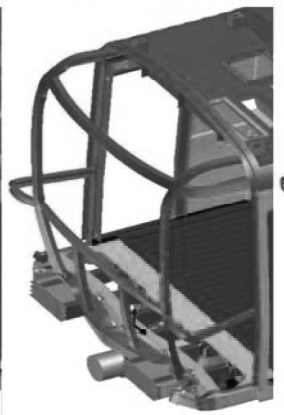

$160 \mathrm{~ms}$

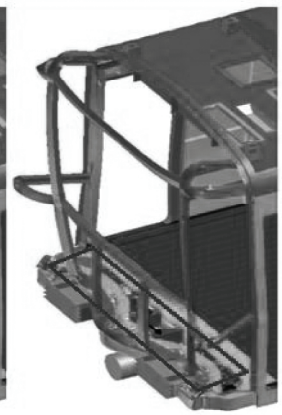

$240 \mathrm{~ms}$
Slight

deformation

Second tube

Opt

Complete

collapse

Second tube

Figure 4. Deformation comparison of initial and optimal design at different moments. 


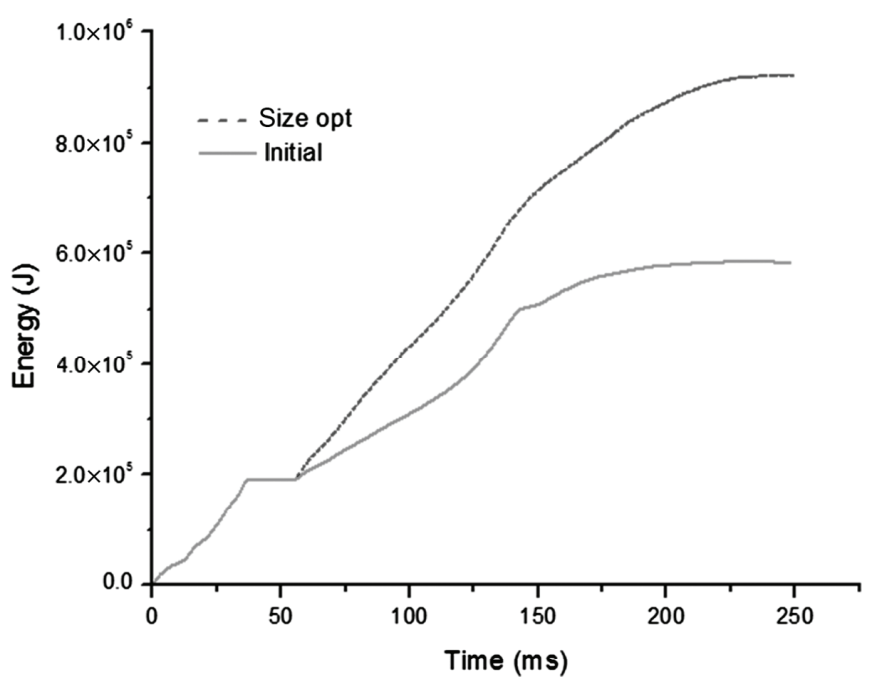

Figure 5. Comparison of total energy absorption.

Table 1

Comparison of Initial Design and Size Optimization Results

\begin{tabular}{|l|c|c|c|}
\hline $\begin{array}{l}\text { First and Second } \\
\text { Structure }\end{array}$ & $\begin{array}{c}\text { Total Energy } \\
(\mathrm{kJ})\end{array}$ & $\begin{array}{c}\text { Mass } \\
(\mathrm{kg})\end{array}$ & $\begin{array}{c}\text { Peak Force } \\
(\mathrm{kN})\end{array}$ \\
\hline Initial & 584.5 & 72.38 & 2,908 \\
\hline Size opt & 922.6 & 62.25 & 2,477 \\
\hline Per cent & 57.8 & 13.9 & 14.8 \\
\hline
\end{tabular}

top is the deformation view of initial design while the bottom is that after size optimization. The first-level tube is collapsed efficiently while there is no same situation appeared with the second level. The deformation of the second level is very slight. It is illustrated that the second-level energy-absorbing tube is not collapsed completely and hardly makes considerable contribution to energy dissipation in promoting crashworthiness of vehicle, which also lead to distinct deformation on the floor in survival space which is not suggested in EN15227 standard.

It is shown in Fig. 5 that energy absorption begins to change after $50 \mathrm{~ms}$ corresponding to the coupler's failure when the energy-absorbing structures enter the collapse state. There is an obvious increase of energy absorption by $57.8 \%$ and a decrease of peak collision force by $14.8 \%$ as listed in Table 1 after size optimization; moreover, the structure mass decreases by $13.9 \%$.

The thickness of the first level increases from 2.0 to $3.56 \mathrm{~mm}$, while the second level decreases from 8.0 to $4.0 \mathrm{~mm}$, in which collision force obviously increased from 70 to $130 \mathrm{~ms}$ and decreased at the second state of 130 230 ms as shown in Fig. 6.

Size optimization has increased energy absorption and decreased peak force during the crash case of vehicle towards a rigid wall, which greatly promotes crashworthiness to some extent and realizes the lightweight design.

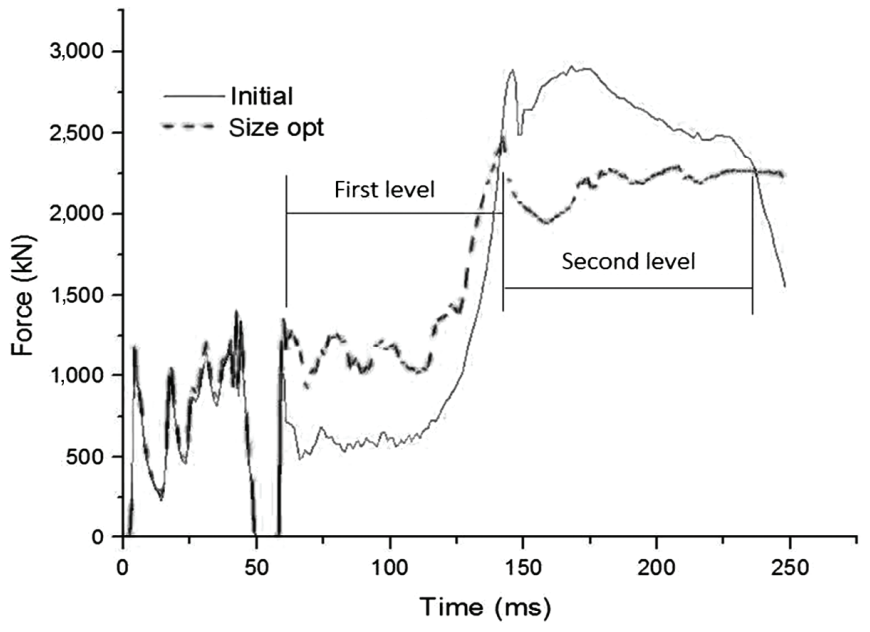

Figure 6. Comparison of collision force.

\subsection{Results Comparison to Response Surface Method}

To validate the results of size optimization by the proposed method mentioned above, an approximate optimization model based on RSM (response surface method) was established to predicate the relation between energy absorption $(E)$ and peak force $(F)$ to parameters of tubes $\left(T_{1}\right.$, $T_{2}$ ), we compared the results by the RSM and the method proposed in this paper. There were 40 sample points generated by the optimal latin hypercube technique in Isight for establishing the RSM model. The response information of the energy absorption and peak collision force is given in the following polynomials (12), (13), where $E$ represents the energy absorption of first-level and second-level tubes, $F$ is the peak of the collision force:

$$
\begin{array}{r}
E\left(T_{1}, T_{2}\right)=47567.19 T_{1}-58493.4 T_{2}-2268.5 T_{1}{ }^{\wedge} 2 \\
+1228.6 T_{2}{ }^{\wedge} 2+8930.1 T_{1}{ }^{*} T_{2}+577002 \\
F\left(T_{1}, T_{2}\right)=369.7 T_{1}+1410.1 T_{2}-11.2 T_{1}{ }^{\wedge} 2 \\
-68.7 T_{2}{ }^{\wedge} 2-61.8 T_{1}{ }^{*} T_{2}-2380
\end{array}
$$

To assess the quality of RSM model established above, random values of variables $\left(T_{1}, T_{2}\right)$ were produced for the error analysis of the approximate model. The closeness degree of results from error analysis points and prediction by RSM model is shown in Fig. 7 . The R-square $\left(R^{2}\right)$ index is adopted and its value for $E$ and $F$ are, respectively, 0.97516 and 0.97001 , each of them exceeds the acceptance level of 0.9 .

Under the objective of maximum energy absorption and minimum peak force, the optimal size parameters of energy-absorbing structure were obtained by solving the RSM model established. The thickness of the first-level tube is $4.00 \mathrm{~mm}$, while the second is $4.04 \mathrm{~mm}$, results comparison are listed in Table 2. 

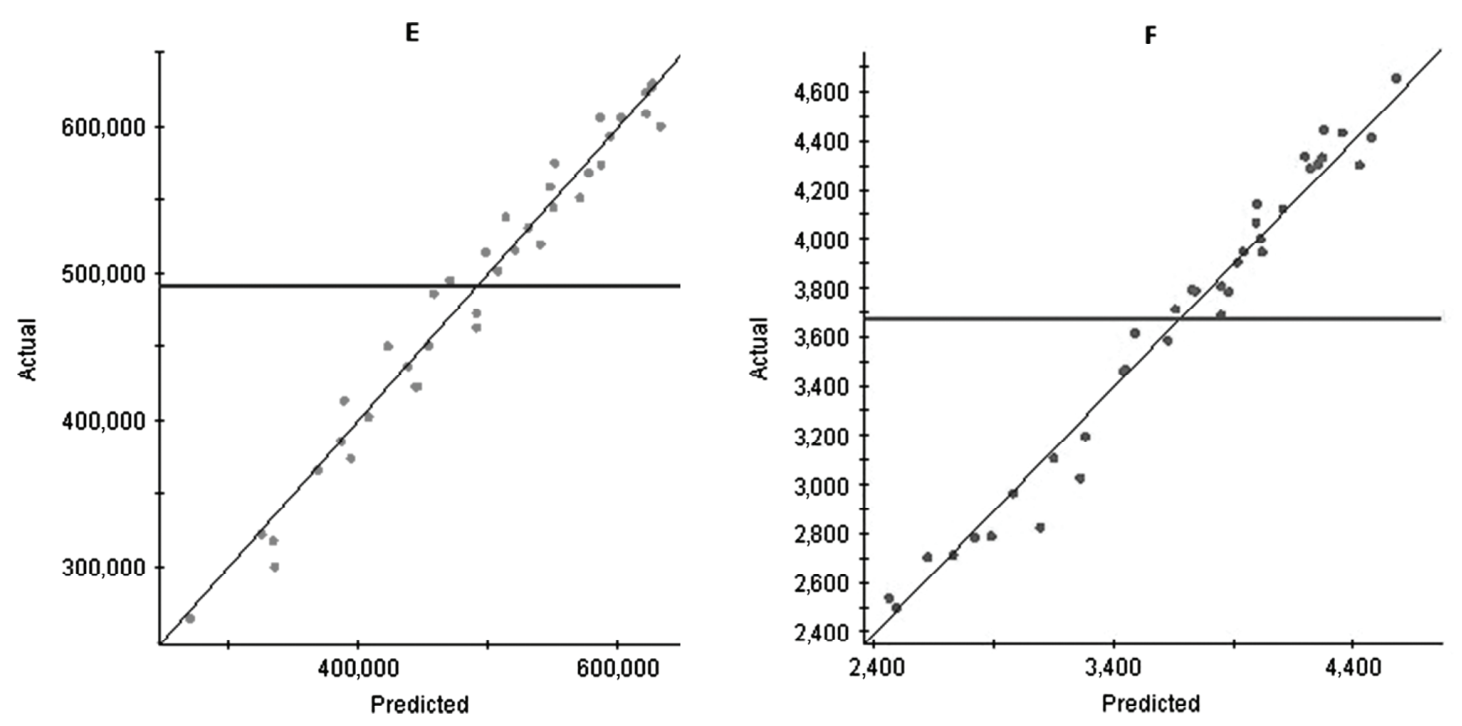

Figure 7. Error analysis of energy $(E)$ and peak force $(F)$.

Table 2

Results Comparison of RSM and Method in This Paper

\begin{tabular}{|l|c|c|}
\hline & $T_{1}(\mathrm{~mm})$ & $T_{2}(\mathrm{~mm})$ \\
\hline Method in this paper & 3.56 & 4.00 \\
\hline RSM & 4.00 & 4.04 \\
\hline
\end{tabular}

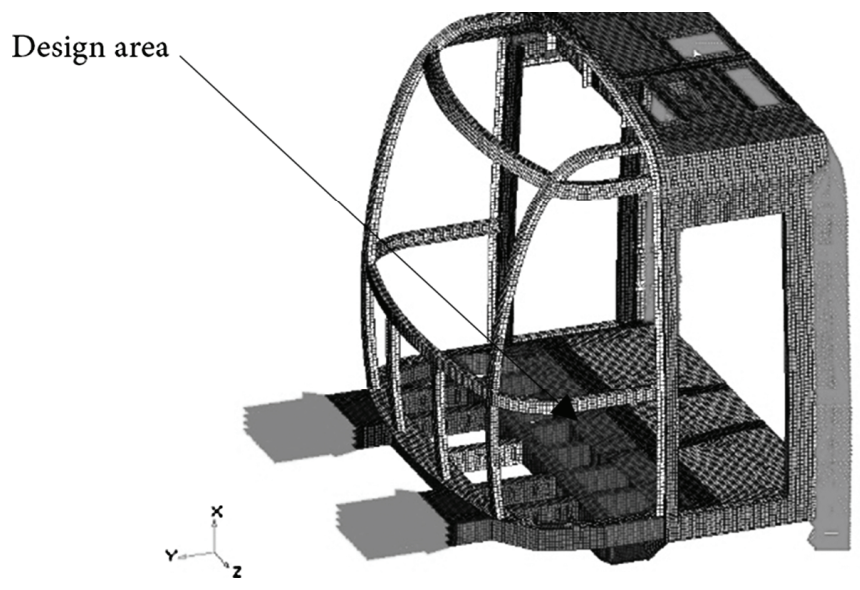

Figure 8. Boundary condition for topology optimization.

\section{Topology Optimization}

\subsection{Design Area and Boundary Condition}

It was indicated that the tubes at the middle position of second level were not collapsed completely as shown in Fig. 4, so topology optimization was put forward to obtain a more efficient force transferring path for second level tube after the size optimization.

The design area of topology optimization in the front end of the vehicle is located at the second-level energyabsorbing tube shown in Fig. 8. The impact load was applied on the nodes of anti-climber, the displacement

constraint was applied on the nodes of car body section marked with red label in Fig. 8.

\subsection{Analysis Results}

As shown in Fig. 9, the area in red colour represents the location with a density value of 1 , while the area in blue colour represents the location with a density of 0 where the material can be removed. Other areas in colours between blue and red represent the density values of 0 to 1 . The colour in topo optimization results just represents reservation or removal of the material applied on structure. The density value in Fig. 9 is the relative density rather than actual physical density, one indication of the load transfer mode, which gives some directions for energy-absorbing component position layout.

It is indicated that collision force is transferred from the side path more than the middle position of second level according to density colour distribution in Fig. 9 (left). Based on the topology optimization result, the tubes at the middle position from the second level of energy-absorbing structure were removed to generate a new optimal structure for energy absorption as shown in Fig. 9 (right).

It is necessary to re-analyse the new design structure by topology optimization shown in Fig. 9 (right) and compare its deformation with that by size optimization in the same load case.

\subsection{Analysis for Redesigned Structure}

To prove the robustness of new structure refined by the optimization result, additional analyses were carried out for vehicle collision at a different speed of 15, 20 and $25 \mathrm{kmph}$. The final deformation results of energy-absorbing structure by size and topology optimization are shown, respectively, in Fig. 10, the deformation of the new design structure by topology optimization appears in a stable and complete manner, especial for the case of 20 and $25 \mathrm{kmph}$, the topology optimization results are feasible and effective. 

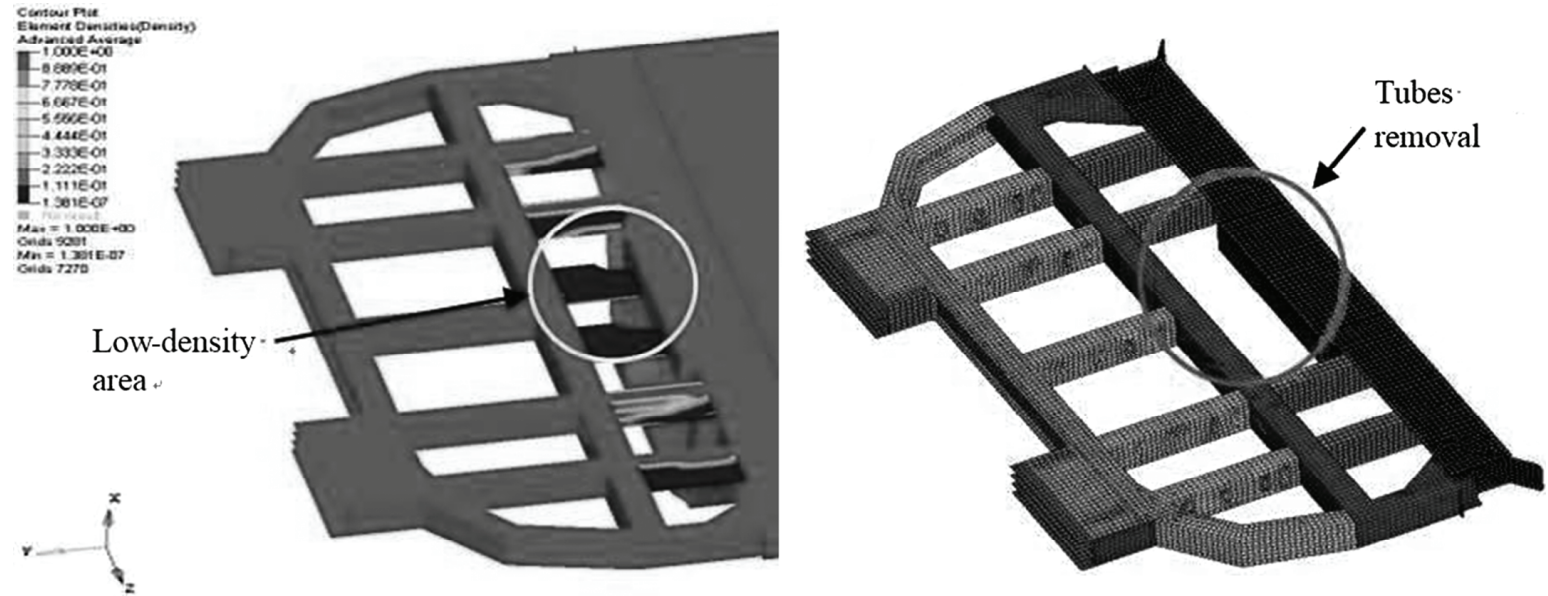

Figure 9. Relative density distribution of topology optimization (left) and redesigned energy-absorbing device (right).
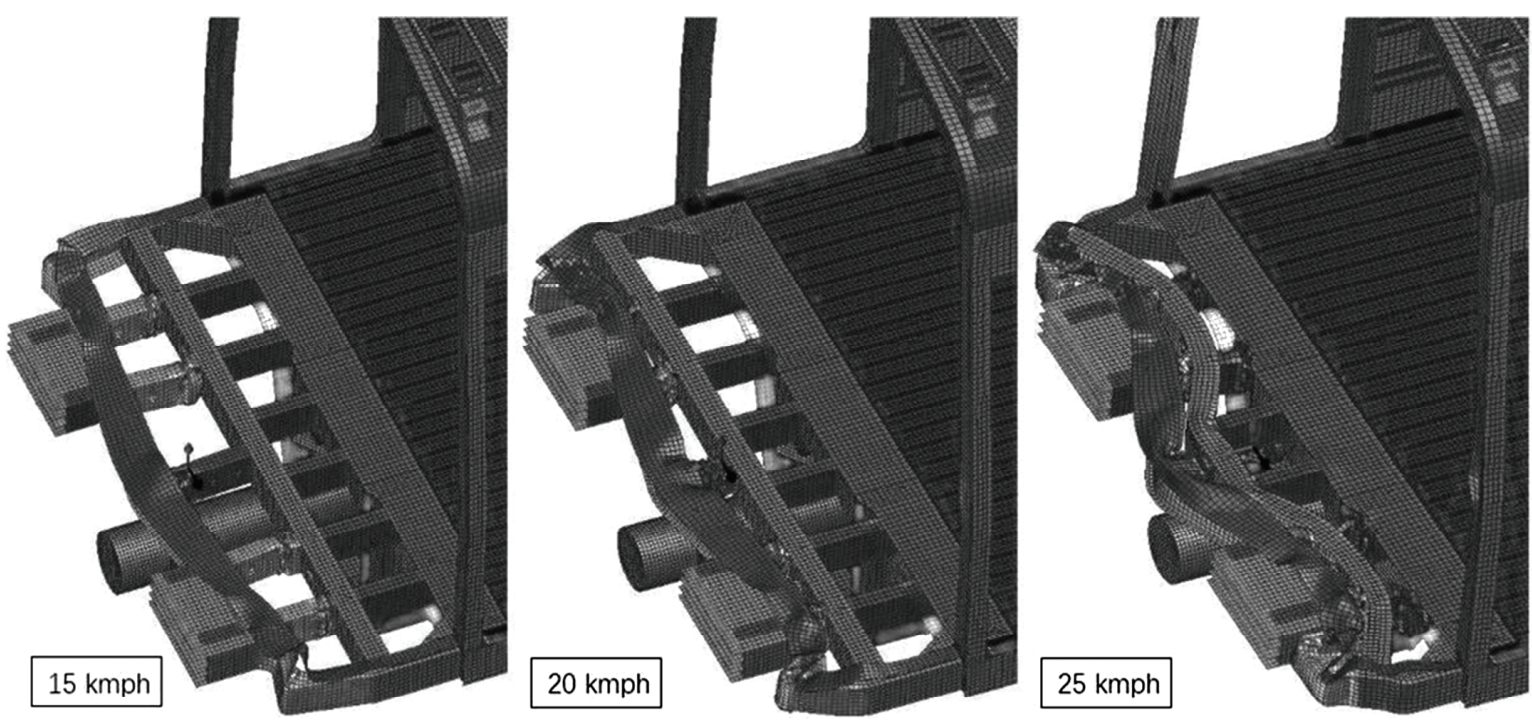

(a)
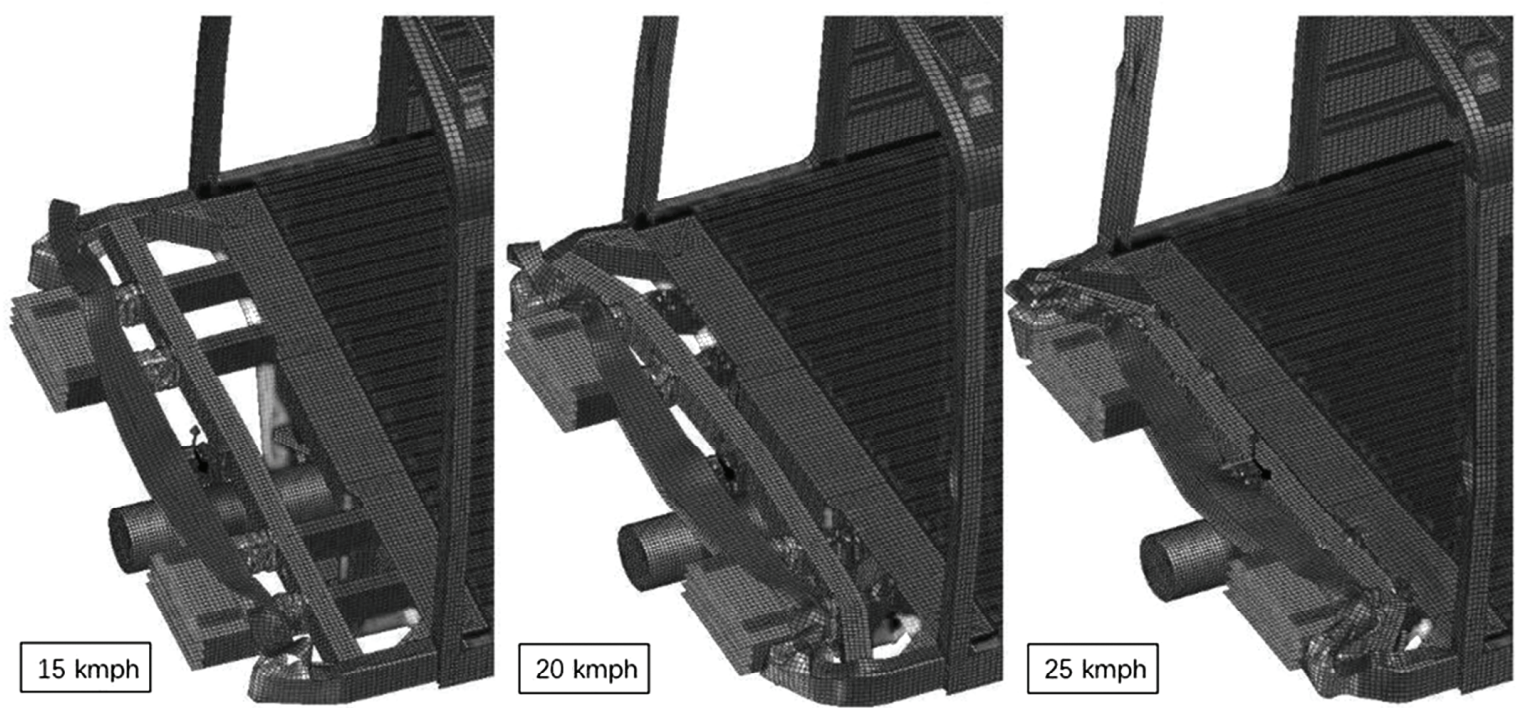

(b)

Figure 10. Deformation comparison before (a) and after (b) topology optimization. 


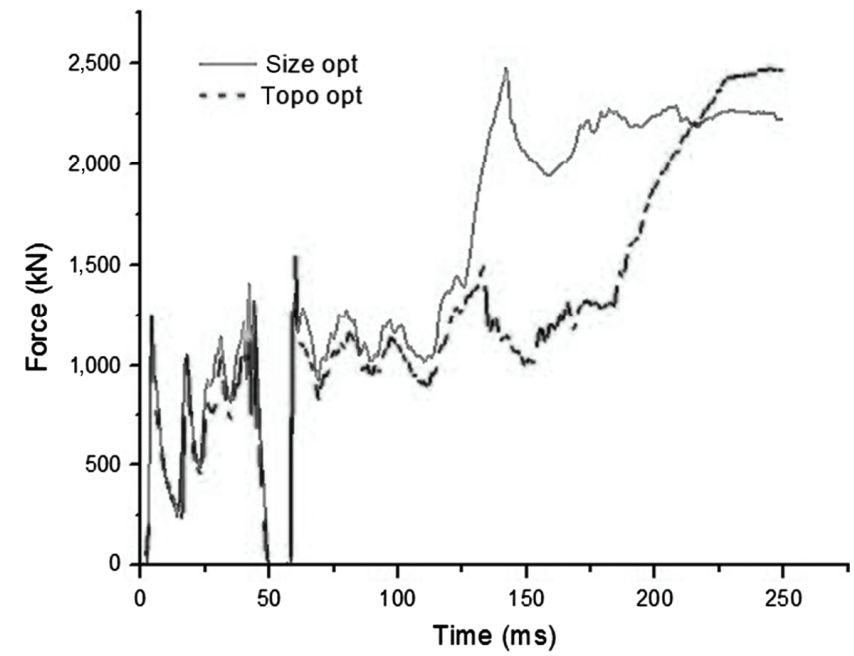

Figure 11. Collision force curves of size and topology optimization.

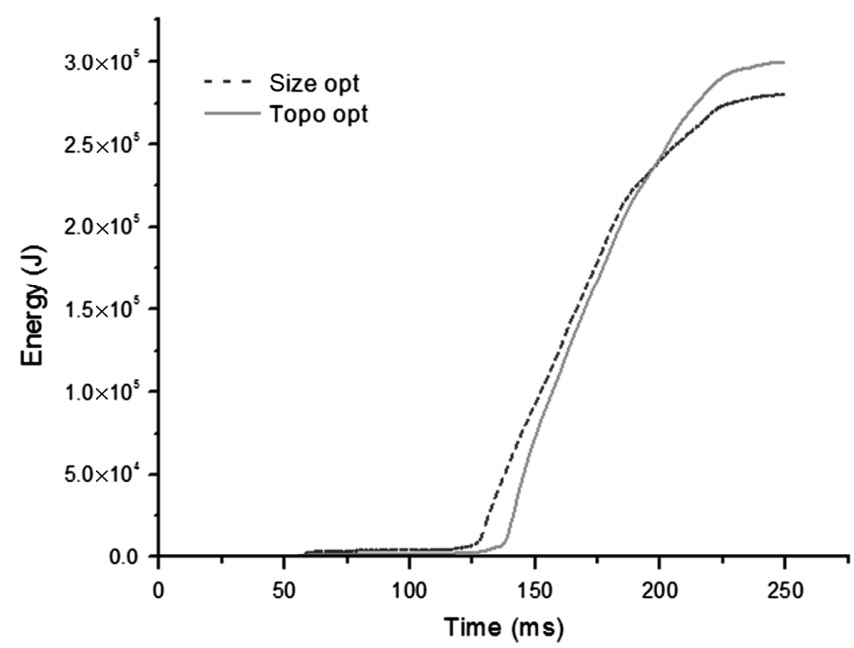

Figure 12. Energy of second-level energy-absorbing structure from topology and size optimization.

It is indicated from the deformation comparison that the energy-absorbing structure was collapsed in a controlled manner with stable deformation mode during collision at different speeds, which meets the requirement of gradual energy dissipation for multi-level structure.

There is an obvious change of collapse force after the first-level tube's complete collapse shown in Fig. 11, which begins to appear approximately at $130 \mathrm{~ms}$.

As a result of complete and sufficient collapse, the energy absorbed by the second-level tube increases $54 \mathrm{~kJ}$ as shown in Fig. 12, but it does not increase total energy absorption revealed from Fig. 13. This can be illustrated from the deformation of two cross girders before and after two energy-absorbing tube's removal shown in Fig. 10, the bending of two cross girders before removal is large than the situation of tube removed. So, the energy increase from the second level is balanced by the decrease from less bending deformation of two cross girders.

Comparison results of topology optimization based on the previous size optimization are summarized in Table 3,

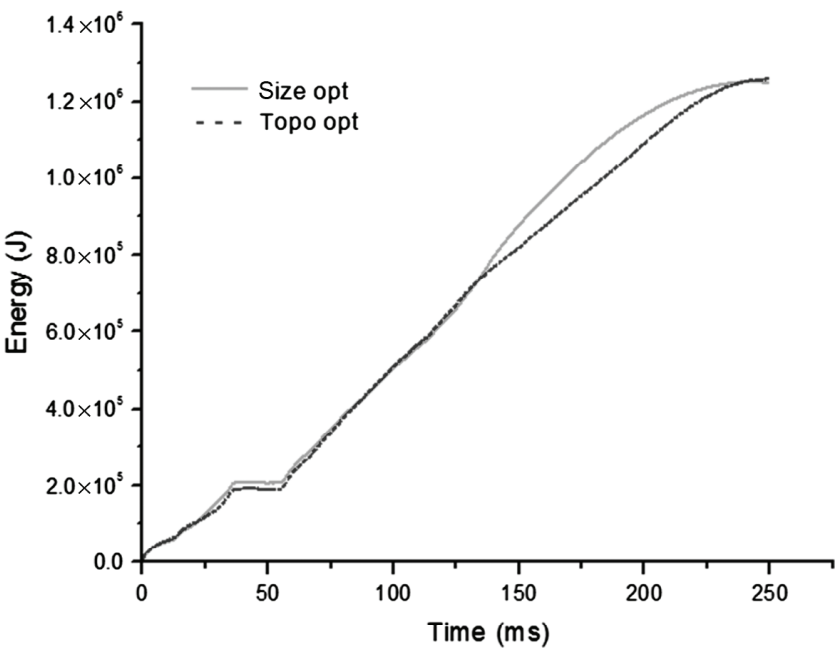

Figure 13. Total energy of size and topology optimization.

Table 3

Comparison of Size and Topology Optimization Results

\begin{tabular}{|l|c|c|c|}
\hline $\begin{array}{l}\text { Second } \\
\text { Structure }\end{array}$ & $\begin{array}{c}\text { Energy } \\
(\mathrm{kJ})\end{array}$ & $\begin{array}{c}\text { Mass } \\
(\mathrm{kg})\end{array}$ & $\begin{array}{c}\text { Peak Force } \\
(\mathrm{kN})\end{array}$ \\
\hline Size opt & 231 & 36.5 & 2,477 \\
\hline Topo opt & 285 & 18.9 & 1,760 \\
\hline Per cent & 23.4 & 48.2 & 28.9 \\
\hline
\end{tabular}

the energy absorbed by the second level increases to 285 $\mathrm{kJ}$ and the peak collision force reduces to $1,760 \mathrm{kN}$, which gives a further promotion to the crashworthiness of vehicle on the basis of size optimization. By adopting topology optimization, a better load transferring path was obtained for improvement of new energy-absorbing design.

\section{Conclusion}

It was successfully realized to solve optimization design for the two-level energy-absorbing tubes of vehicle by combining the integration platform Isight with the explicit dynamical analysis code PAM-CRASH. Furthermore, the topology optimization was applied to determine the load transfer path for the second-level tubes of energy-absorbing structure. Both the promotion of crashworthiness and lightweight design of energy-absorbing tube were realized. It was revealed that the optimization strategy combing multi-aspects is capable and efficient in improving the passive safety for the railway vehicle during a collision, it is also suitable for other engineering structure.

\section{Acknowledgement}

This research was supported by the National Natural Science Foundation of China (Project No. 11272070) and Research plan on science and technology of China Railway Corporation (Project No. 2015J007-H) and the National Social Science Foundation of China (Grant No. 13BGL143). 


\section{References}

[1] Technical Committee CEN/TC 256 Railway Applications, BS EN 15227-2008 Railway Applications: Crashworthiness Requirements for Railway Vehicle Bodies, British Standard Institution, London, 2008.

[2] Railtrack. Railway Group Standard GM/RT2100 Structural Requirements for Railway Vehicles, British Standard Institution, London, 2000.

[3] T. Belytschko, R.E. Welch, and R.W. Bruce, Finite element analysis of automotive structures under crash loadings, Proc. IIT Research Institute, Chicago, IL, 1975, 37-46.

[4] H. Kurtaran, A. Eskangarian, D. Marzougui, et al., Crashworthiness design optimization using successive response surface approximations, Computational Mechanics, 29(4-5), 2002, 409-421.

[5] H.R. Zarei and M. Kröger, Multiobjective crashworthiness optimization of circular aluminum tubes, Thin-Walled Structures, 44(3), 2006, 301-308.

[6] S. Xie, X. Liang, H. Zhou, et al., Crashworthiness optimisation of the front-end structure of the lead car of a high-speed train, Structural and Multidisciplinary Optimization, 53(2), 2015, 339-347.

[7] X. Gu, G. Sun, G. Li, et al., A comparative study on multiobjective reliable and robust optimization for crashworthiness design of vehicle structure, Structural and Multidisciplinary Optimization, 48(3), 2013, 669-684.

[8] F. Jiazhi, J. Qunying, C. Chongyi, et al., Application of response surface method in impacting energy absorption of thin-walled structure, Journal of China Agricultural University, 1, 2015, 021 .

[9] L. Lanzi, L.M.L. Castelletti, and M. Anghileri, Multi-objective optimization of composite absorber shape under crashworthiness requirements, Composite Structures, 65, 2004, 433-441.

[10] G. Sun, X. Song, S. Baek, et al., Robust optimization of foam-filled thin-walled structure based on sequential Kriging metamodel, Structural and Multidisciplinary Optimization, 49(6), 2014, 897-913.

[11] M. Mrzygłód and T. Kuczek, Uniform crashworthiness optimization of car body for high-speed trains, Structural and Multidisciplinary Optimization, 49(2), 2014, 327-336.

[12] H.J. Kim, H. Cho, H.S. Jung, et al., Crashworthiness design and evaluation on the leading-cab structure of rolling stock using topology optimization, International Journal of Precision Engineering and Manufacturing, 10(2), 2009, 79-85.

[13] C.B. Pedersen, Topology optimization design of crushed 2Dframes for desired energy absorption history, Structural and Multidisciplinary Optimization, 25(5-6), 2003, 368-382.

[14] R.R. Mayer, N. Kikuchi, and R.A. Scott, Application of topological optimization techniques to structural crashworthiness, International Journal for Numerical Methods in Engineering, 39(8), 1996, 1383-1403.

[15] C.B.W. Pedersen, Topology optimization design of crushed 2D-frames for desired energy absorption history, Structural and Multidisciplinary Optimization, 25(5-6), 2003, 368-382.

[16] M. Avalle, G. Chiandussi and G. Belingardi, Design optimization by response surface methodology: Application to crashworthiness design of vehicle structures, Structural and Multidisciplinary Optimization, 24(4), 2002, 325-332.

[17] D. Spath, W. Neithardt, and C. Bangert, Integration of topology and shape optimization in the design process, CIRP, 4, 2001, 4.

[18] T. Zhang, M. Wu, Y. Zhao, et al., Optimal motion planning of mobile welding robot based on multivariable broken line seams, International Journal of Robotics and Automation, 29(2), 2014, 215-223.

[19] X. Chen, J. Wu, L. Wang, et al., Optimum design of a parallel conveyor for electrocoating of vehicle bodies, International Journal of Robotics and Automation, 31(3), 2016, 198-205.

[20] L. Liang, P. Tang, B. Chen, et al., Dynamical modelling and structural parameter optimization of a novel spiral inpipe robot, International Journal of Robotics and Automation, 31(1), 2016, 19-28.
[21] K. Schittkowski, NLPQL: A FORTRAN subroutine solving constrained nonlinear programming problems, Annals of $O p$ erations Research, 5(1), 1986, 485-500.

[22] M.P. Bendsøe, Optimal shape design as a material distribution problem, Structural Optimization, 1(4), 1989, 193-202.

\section{Biographies}

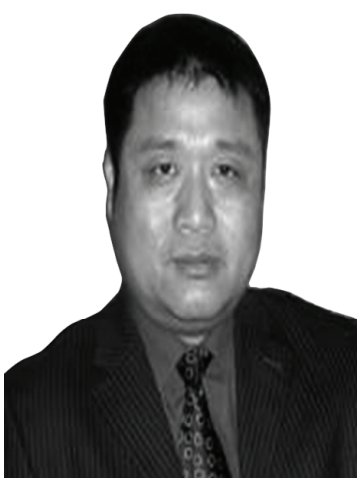

Bingzhi Chen is a professor and Ph.D. supervisor at Dalian Jiaotong University of China. $\mathrm{He}$ is Dean of the School of Traffic and Transportation Engineering in Dalian Jiaotong University and also an academic leader of the Vehicle Structure Design and Collaborative Optimization. His main research interests include railway vehicles engineering, virtual prototype, numerical simulation, structure optimization and biomechanics. In the recent five years, he has been in charge of several research projects, with two projects on National Natural Science Funds, two projects funded by Ministry of Railways, one by National Post-doctoral Fund and one by National Science and Technology Support Program. He has completed one of the National 863 Project.

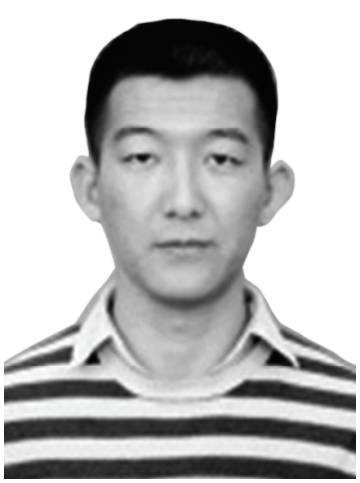

Ruixian Qin is a Ph.D. degree candidate from Dalian Jiaotong University of China. His main research interests include passive safety and optimization of railway vehicles. He has participated and completed several projects involving crashworthiness simulations for railway vehicles and key projects supported by National Natural Science Funds.

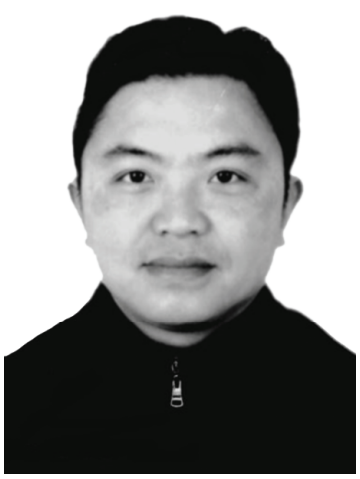

Chaozhe Jiang is an associate professor of both Transportation Engineering and Management Science at Southwest Jiaotong University. Currently, he is a research associate of the Department of Civil and Environmental Engineering at University of Waterloo, Canada. He joined Southwest Jiaotong University as postdoctoral researcher in transportation engineering until 2009. $\mathrm{He}$ joined the Department of Civil and Environmental Engineering, University of Waterloo, Canada as post-doctoral research since 2010. His research interests include Intelligent Control and Decision Making, Comprehensive Transportation Planning, Rough Set Theory, Transportation Systems Analysis and Engineering, Urban Traffic PM2.5 Emission Modelling, and Railway Marshalling Station Scheduling, Big Data Application in Transportation. 